\title{
The effect of earth tides as observed in seismo-electromagnetic precursory signals
}

\author{
M. Hayakawa ${ }^{1}$, Y. Sue ${ }^{2}$, and T. Nakamura ${ }^{3}$ \\ ${ }^{1}$ The University of Electro-Communications (UEC), Advanced Wireless Communications Research Center and Research \\ Station on Seismo Electromagnetics, 1-5-1 Chofugaoka, Chofu Tokyo 182-8585, Japan \\ ${ }^{2}$ Private scientist, 3-14-14 Nakaochiai, Shinjuku-ku, Tokyo 161-0032, Japan \\ ${ }^{3}$ UEC, Department of Electronic Engineering, Chofu Tokyo, Japan
}

Received: 24 July 2009 - Accepted: 30 September 2009 - Published: 27 October 2009

\begin{abstract}
Studies on the effect of earth tides in triggering earthquakes (EQs) had a great progress in recent years, which has provided convincing evidence of earth tides in EQ triggering. On the other hand, there have been accumulated a lot of evidences on the presence of seismogenic electromagnetic effects (such as ULF electromagnetic emissions from the lithosphere, ionospheric perturbations as detected by subionospheric VLF/LF propagation, etc.). Since the initial agent of these seismogenic electromagnetic effects is obviously due to some mechanical action around the EQ focal zone, the tidal effect as seen in EQ sequence should appear also in seismo-electromagnetic phenomena. Based on this expectation we have studied the tidal effect in different seismogenic phenomena, and have found that lithospheric ULF emissions exhibit a clear maximum-minimummaximum pattern synchronized with the lunar phase of the EQ during several months before the EQ. As for VLF/LF propagation anomaly representing the lower ionospheric perturbation, we have found the tidal modulation very similar to ULF emissions, but less clear, and also there are some differences from the ULF case (such as occasional shift with respect to the lunar phase and/or the presence of higher frequency modulation, etc.). These findings are indicative that those electromagnetic phenomena reported to be in possible association with an EQ are really related with any preparatory phase of an EQ. This kind of study would be a bridge between the seismology and our seismo-electromagnetic study.
\end{abstract}

\section{Introduction}

The Earth travels around the Sun as a planet in the solar system, and the Moon orbits the Earth as a satellite. These

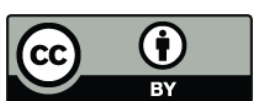

Correspondence to: M. Hayakawa (hayakawa@whistler.ee.uec.ac.jp) movements are known to create changes of the seasons as well as changes in the apparent shape of the Moon, so that these movements also affect seawater, which is called the ocean tide. The same mechanism is applied to the Earth's body, and the solid Earth deforms like seawater. This phenomenon is called the earth tide (or lunar-solar tide), with which the Earth's surface moves like the surface of the sea, some ten centimeters vertically. At the same time the Earth's crust which covers the Earth's surface, is expanded, contracted and inclined, as is schematically illustrated in Fig. 1. It has been debated for years whether earth tides trigger EQs. A recent paper by Tanaka et al. (2004) has been published on a statistical study of tidal triggering, which has proven the significance of these earth tides in EQ triggering. Following the study, Tanaka (2006) has provided some more evidences that the EQs occurred near the epicenter of the 2004 Sumatra EQ were affected by earth tides for the period of 10 years before the main shock. Sue (2009) has then shown the evidences of apparent tidal effects in EQ triggering in the 3 specific regions in Japan such as the Sagami trough region by paying particular attention to some specific fault structures.

EQs are definitely a mechanical phenomenon, so that it is not so difficult to imagine that the earth tide is a possible candidate of triggering EQs when the focal zone is selforganized to a critical or a super-critical stage. On the other hand, there have been accumulated recently a lot of evidences on the presence of precursory electromagnetic effects of EQs (Hayakawa, 1997, 1999; Hayakawa and Molchanov, 2002, 2007; Molchanov and Hayakawa, 2008). We can list a few examples. The ULF (ultra-low-frequency) electromagnetic emissions as the 1st example are believed to be generated near the EQ focal zone and to have propagated up to the Earth's surface (e.g., Fraser-Smith et al., 1990; Kopytenko et al., 1993; Hayakawa et al., 1996a). Molchanov and Hayakawa $(1995,1998$ a) proposed a mechanism of microfracturing for seismogenic ULF emissions. Whatever the mechanism is (microfracturing or electrokinetic), these

Published by Copernicus Publications on behalf of the European Geosciences Union. 

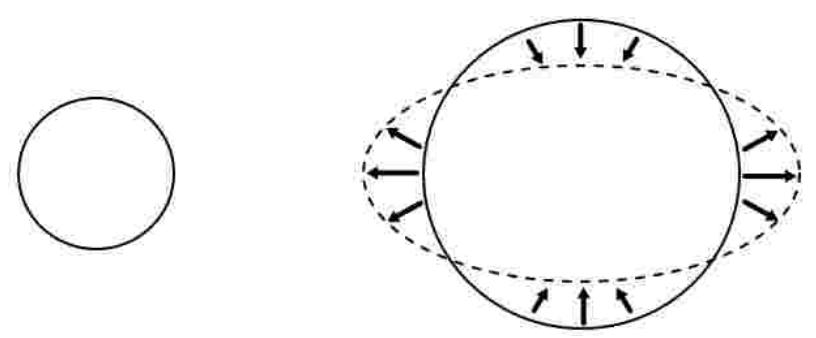

Moon or Sun

Earth

Fig. 1. Schematic picture of earth tides (expansion, contraction, inclination).

ULF emissions are fundamentally based on any mechanical effect in the EQ focal zone. The next phenomenon clearly associated with EQs is the ionospheric perturbations(e.g. Hayakawa et al., 1996b; Hayakawa, 2007). Especially the lower ionosphere (D/E region) is found to be very sensitive to EQs, as based on the use of subionospheric VLF/LF propagation characteristics. The mechanism how and why the ionosphere is perturbed prior to an EQ, is not well understood, though we have proposed a few hypotheses (Hayakawa, 2004; Molchanov and Hayakawa, 2008); (i) chemical (and electric field effect) channel, (ii) acoustic and gravity wave channel and (iii) electromagnetic channel. No matter which mechanism is most plausible, the initial effect of seismogenic phenomena is located near the EQ focal region and it is just a mechanical effect (not macroscopic, but probably microscopic).

So that, the purpose of this paper is to try to find out any modulation of earth tides in various kinds of seimogenic phenomena (such as seismogenic ULF emissions, seismogenic ionospheric perturbations, etc.). This study would be a bridge between the seismology and our seismoelectromagnetics, and also this effect would offer an important additional confirmation on the presence of seismogenic electromagnetic effects.

\section{Analysis method}

The earth tides of the Moon and the Sun affect the occurrence of EQs. This is the phenomenon which appears only when earth tides deform a fault from the direction to assist the fault slipping (and the corresponding seismogenic electromagnetic effects). Thus we investigate if there are any affections of the specific lunar phase in electromagnetic EQ precursors.

The lunar phase is explained in Fig. 2. For an index of the lunar phase, it is possible to apply the ecliptic longitude difference between the Moon and the Sun of the ecliptic coordinate system. This is the angle with a numerical value of 0 to $360^{\circ}$ : new moon at $0^{\circ}$, first quarter moon at $90^{\circ}$, full

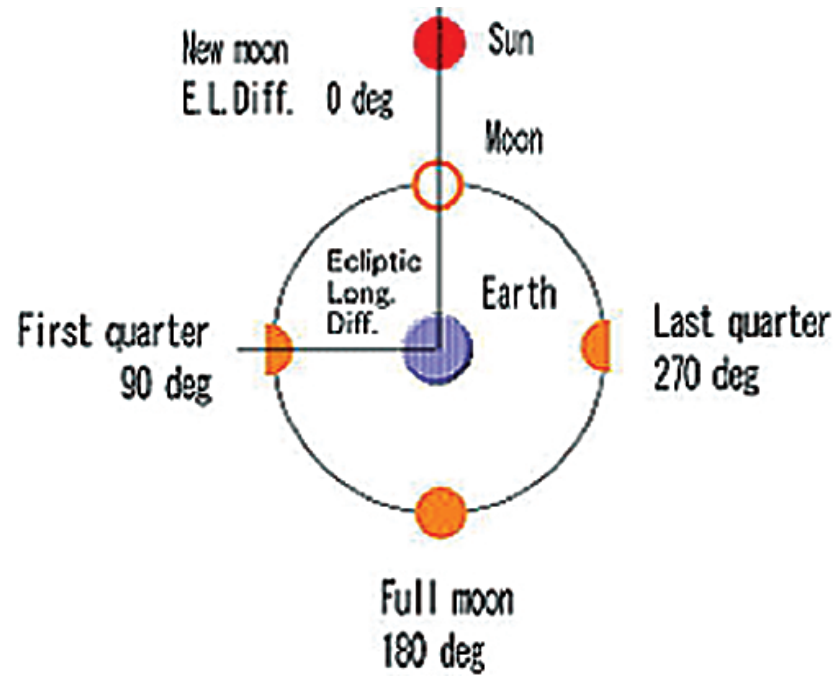

Fig. 2. Explanation of lunar phase $\left(0^{\circ}\right.$, new moon, $90^{\circ}$, first quarter, $180^{\circ}$, full moon and $270^{\circ}$, last quarter).

moon at $180^{\circ}$, and last quarter moon at $270^{\circ}$. Since the Moon orbits the Earth in 29.5 days on average, so that the average traveling speed of the Moon is $12.2^{\circ}$ per day. In this paper, we use the number of days with respect to such specific lunar positions as the new, first quarter, full and last quarter moon as the indicator of lunar phase.

\section{The effect of earth tides as observed in seismo-electromagnetic precursory signals}

We investigate the effect of earth tides in different phenomena of ULF geomagnetic anomalies, VLF propagation anomalies, and radio emission anomalies at different frequencies. We study these phenomena one by one in the following.

\subsection{ULF geomagnetic anomalies}

The reports of ULF geomagnetic anomalies before large EQs have been summarized in Hayakawa et al. (2007). The ULF signal observed at any particular station is known to be composed of different noise sources including the dominant geomagnetic variation (geomagnetic pulsations), artificial noise and seismogenic noise as our target. So that, it is very difficult to find any relationship to the lunar phase when we use the raw data, and it is of essential importance to perform any signal pre-processing to the raw data to distinguish our seismogenic noise from the whole signal or to enhance our seismogenic signal against the other noises. There have been developed a few possible methods for this purpose: the first is called "polarization" analysis, in which the use of the ratio of the vertical $(\mathrm{Z})$ to horizontal $(\mathrm{H})$ magnetic field components allows us to identify the seismogenic effect against the space 
noise (geomagnetic variation) (Hayakawa et al., 1996a). The second is the use of principal component analysis (PCA), in which a multiple of stations of simultaneous observation enables us to distinguish a few possible noise sources (such as (1) geomagnetic variation, (2) man-made noise, and (3) seismogenic emission) (Gotoh et al., 2002).

One more important point to mention as regards the seismogenic ULF emissions is their typical temporal evolution. They show the first peak one to two weeks before the EQ, become quiet in amplitude several days before the EQ and exhibits an abrupt increase a few days before the EQ (FraserSmith et al., 1990; Kopytenko et al., 1993; Hayakawa et al., 1996a).

We here deal with our former two ULF events; the 1993 Guam EQ and the 2000 Izu islands EQ swarm in the following.

\section{Case 1: the 1993 Guam EQ $(M=8.0)$}

The Guam EQ ( $M=8.0$, Depth $59 \mathrm{~km})$ occurred on 8 August 1993 (Hayakawa et al., 1996a). ULF geomagnetic anomalies were observed for this EQ, but the relationship with earth tides could not be identified in the raw data due to the dominant contribution by the geomagnetic variation.

With the polarization analysis shown in Fig. 3, the ratio of $\mathrm{Z} / \mathrm{H}$ has exhibited the following characteristics (Hayakawa et al., 1996a). The main point of Hayakawa et al. (1996a) is that we observed an enhanced $\mathrm{Z} / \mathrm{H}$ during one month before the EQ, which is likely to reflect the occurrence of seismogenic emissions. The enhanced $\mathrm{Z} / \mathrm{H}$ lasts for about one month, so that we take the EQ day as a reference point, 3 days before the last quarter moon as shown as LQ-3d in the figure. The days of the same lunar phase are marked $(-1 \mathrm{~m}$, $-2,-3$, and -4 ) for the period of 4 months before the EQ in the figure. We note that the period between the same lunar phases is 29.5 days on average (a lunar month), which is the duration of the moon's orbiting the Earth. The value of $\mathrm{Z} / \mathrm{H}$ is increased 2 months before the EQ, further it takes a maximum (at $-1 \mathrm{~m}$ ) at the same lunar phase as that of the EQ and minima (shown in broken lines) in the middle. There are several small oscillations with the different lunar phases, suggesting overlapping of other patterns with different lunar phases. As the summary, the behavior of $\mathrm{Z} / \mathrm{H}$ shows a clear maximum-minimum-maximum pattern synchronizing with the lunar phase of the EQ during 2 months before its occurrence.

\section{Case 2: the 2000 Izu islands EQ swarm $(M=6.4$, etc. $)$}

From June to August in 2000 there was observed a series of large tectonic activities which included a total of more than 3000 EQs with 5 large EQs (July 1: $M=6.4$, July 9: $M=6.1$, July 15: $M=6.3$, July 30: $M=6.4$ and August $18: M=6.0$ ) and 5 eruptions at Miyake island (Toda et al., 2002). This activity was caused by intrusion of magma and such EQ activities

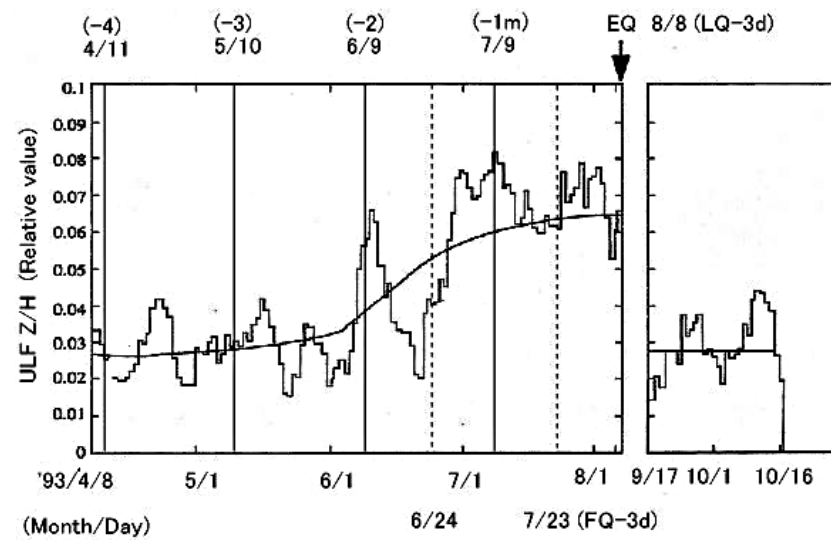

Fig. 3. Lunar phase analysis for the polarization $(\mathrm{Z} / \mathrm{H})$ of ULF geomagnetic emissions (at the frequency of $0.01 \mathrm{~Hz}$ ) for the 1993 Guam EQ $(M=8.0)$.

are the response to the stress rate. The ULF electromagnetic radiation has already been shown to indicate anomalies for this activity (Gotoh et al., 2002; Uyeda et al., 2002). The PCA method by Gotoh et al. (2002) enabled us to separate a few noise sources. The 1st principal component (whose eigenvalue is $\lambda 1$ ) is found to correspond very well to the geomagnetic variation, the 2 nd principal component $(\lambda 2)$ is then found to reflect the human activity, so that the 2 nd component is due to the man-made noise. The last and the weakest principal component $(\lambda 3)$ might include the seismogenic effect if it exists. The upper panel of Fig. 4 shows the temporal evolution of the 3 rd principal (weakest) component $\lambda 3$ at the frequency of $0.01 \mathrm{~Hz}$ from 1 February to 16 July 2000, which is taken from Gotoh et al. (2002). The largest EQ ( $M=6.4)$ of the activity occurred on 1 July in Fig. 4, and this day is taken as a reference point corresponding to a new moon 1 day which is shown as $\mathrm{N}-1 \mathrm{~d}$ in the figure. The days of the same lunar phase as well as the envelope curves connecting peaks are indicated for the period of 5 and 3 months respectively. From the beginning of April which is 3 months before the EQ, there are apparent increases of the eigenvalue $\lambda 3$ of the 3 rd principal component at the same lunar phase as that of the EQ and the decreases in the middle. Note that the geomagnetic activity Ap shown in the lower part of Fig. 4, does not show such a tendency. In summary, the behavior of $\lambda 3$ shows a maximum-minimum-maximum pattern synchronizing with the lunar phase of the EQ during 3 months before its occurrence. In addition to the phenomena mentioned, there was another large EQ of $M=6.4$ on 30 July whose lunar phase is again a new moon- 1 day. The next large EQ of $M=6.3 \mathrm{oc}-$ curred on 15 July or a full moon - 1 day, which is the opposite phase of the reference day. Since these activities were caused by intrusion of magna which behaved as fluid, the activities could be high at a new and a full moon like oceans. 


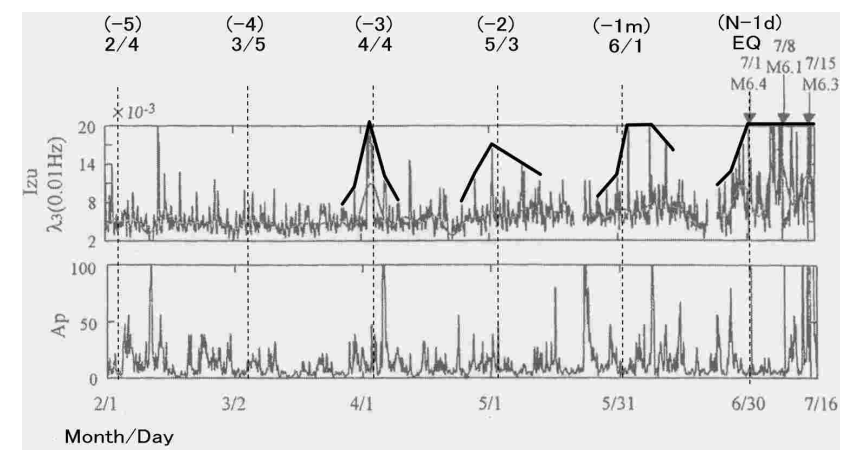

Fig. 4. Lunar phase analysis of the third principal component (its eigenvalue, $\lambda 3$ ) of geomagnetic variation at the frequency of $0.001 \mathrm{~Hz}$ (upper panel) and geomagnetic activity, Ap index (lower panel) for the 2000 Izu islands EQ swarm $(M=6.4$, etc.).

\subsection{VLF propagation anomaly}

There are numerous VLF (in the frequency range in 10$30 \mathrm{kHz}$ ) transmitter stations around the world, and the best known among them is the Omega station. VLF Omega radio waves have been mainly used for navigation, but propagation anomalies before EQs by means of these VLF waves were reported in 1980's and early 1990's, including the 30 December 1983 Caucasus EQ $(M=7.2)$ (Gufeld et al., 1992), 7 December 1988 Spitak EQ in Armenia $\left(M_{s} \sim 7.0\right)$ (Gufeld et al., 1994) and EQs of Japan (Hayakawa and Sato, 1994). The studies on detecting EQ precursors by using different VLF radio transmitters had a great progress during the last decade, and under this situation the Kobe EQ $(M=7.3)$ occurred on 17 January 1995. Figure 5 shows the temporal evolution of the signal received at the Inubo observatory transmitted from Tsushima VLF Omega station (Hayakawa et al., 1996b). Before the EQ, Hayakawa et al. (1996b) observed clear shifts in terminator times (TTs) (defined as the times when the amplitude (and/or phase) of VLF signal exhibits a minimum just around the sunrise and sunset). They interpreted this shift in TTs in terms of the lowering of the lower ionosphere by a few kilometers by using the full-wave theory (Molchanov et al., 1998). While, Yoshida et al. (2008) have treated the same problem by means of wave-hop theory, who estimated how much the lower ionosphere goes down before the EQ.

\section{Case 1: the 1995 Kobe earthquake $(M=7.3)$}

Figure 5 shows the temporal evolutions of TT phase (upper panel) and amplitude (lower panel) for a period of 1.5 months before and after the Kobe EQ, which is taken from Hayakawa et al. (1996b). The upper figure shows " $\Delta t e, p h$ ", which is the variation of the evening TT (phase), where the standard deviation $\sigma$ is that for one month period. The figure shows that on 14 January which is 3 days before the EQ, there was a large deviation exceeding $2 \sigma$. The deviation exceeding $2 \sigma$

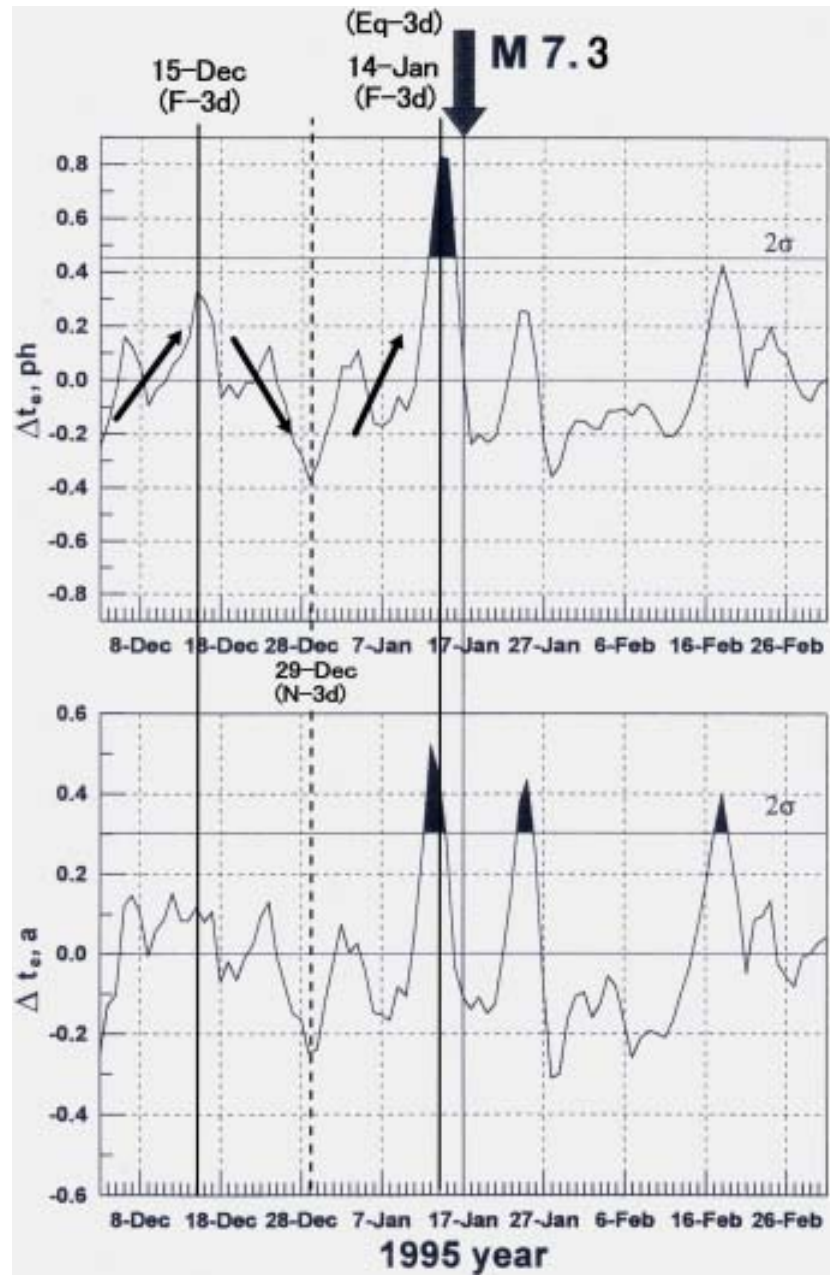

Fig. 5. Lunar phase analysis of the evening TT (phase) (upper panel) and TT (amplitude) (lower panel) for the Kobe EQ $(M==7.3)$. The deviation in TT (evening) ( $\Delta t e)$ exceeding $2 \sigma$ is considered as anomaly (in black). The VLF anomaly takes place on 14 January (EQ-3d and F-3d), and the day with the same lunar phase is also given by a vertical thick line. A vertical dotted line indicates the opposite lunar phase (N-3d).

is an unusual phenomenon, while there were no reports of so extensive geomagnetic activity, solar activity and rainfall in the Kobe region as to affect the VLF transmission (see Hayakawa et al., 1996b; Molchanov et al., 1998). Thus the phenomenon was found to be likely to be a precursory signal of the EQ occurred immediately after (Hayakawa et al., 1996b; Molchanov et al., 1998).

The day of shift in TT exceeding $2 \sigma$ is chosen as a reference point in Fig. 5. The day was 14 January (3 days before the EQ) which was 3 days before a full moon day and is shown as F-3d in the figure. The same lunar day of one month before was 15 December (shown by a vertical thick line) and the day of the middle between the two (shown by a vertical dotted line) which is a new moon -3 days, was 
29 December. The value $(\Delta t e)$ firstly took a maximum at 3 days before a full moon (F-3d), then decreased to take a minimum at 3 days before a new moon $(\mathrm{N}-3 \mathrm{~d})$, and raised again to take a maximum at 3 days before the EQ. And it finally decreased to zero, then the EQ occurred. During this period, we observe some oscillations with the period of 910 days on the basis of spectrum analysis. The deviation from the average increased with time, so the last maximum was the largest and was regarded as a conspicuous precursory to the EQ. The lunar phases at the maximum and minimum in $\Delta t e$ are found to coincide or to be exactly opposite with that of the reference point. We conclude that there was detected a clear maximum-minimum-maximum pattern during one month before the EQ, and the deviation seems to increase with time toward the EQ (thus the last maximum was the largest). After passing the last maximum, the values of $\Delta t e$ decreased (or relaxed) and several days later the EQ occurred when the deviation became nearly zero.

The lower part of Fig. 4 which is " $\Delta t e, a$ ", the amplitude instead of phase also shows the similar patterns as the upper panel.

\section{Case 2: the 1978 earthquake $(M=7.0)$}

After having identified a clear propagation anomaly for the Kobe EQ, Molchanov and Hayakawa (1998b) further studied 11 cases including the Kobe EQ observed for the same combination of transmitter and receiver during 13 years, and obtained the result that when the EQ epicenter was located close to the transmission path, and also when the depth of epicenter was shallow, then $70-80 \%$ of them showed anomalies before an EQ. We have performed the similar analysis of the tidal effect in the VLF data in Molchanov and Hayakawa (1998b) as done above. Those events are not found to show such a strict relationship as for the Kobe EQ (mentioned above), but all cases are found to show a possible relationship with earth tides. We indicate the results of these further studies.

Figure 6 refers to the evening TT (phase) $(\Delta t e, p h)$ of the VLF propagation for the relevant EQ. There are anomalies for which $\Delta t e$ exceed $2 \sigma$ twice before the EQ (as shown by black area), and the first (in time) anomaly is chosen as a reference point of the analysis. The anomaly appeared 10 days before the EQ and the day was a last quarter moon +2 days which is shown as LQ+2d in the figure. The day of same lunar phase of one month before, and the day between the two which is the day of a first quarter moon +2 days are indicated in the figure.

It seems from the figure that the result does not show any strict relationship with the lunar phase, but a day near 1 month before the reference point it took a maximum, then decreasing with passing through 2 small peaks and bottoms, taking a minimum, then it increased again and exceeded $2 \sigma$. Then $\Delta t e$ showed an oscillation with period of 14-15 days (one half of the lunar period) and 7-8 days, finally decreased and reached the EQ day at the deviation around 0 . The

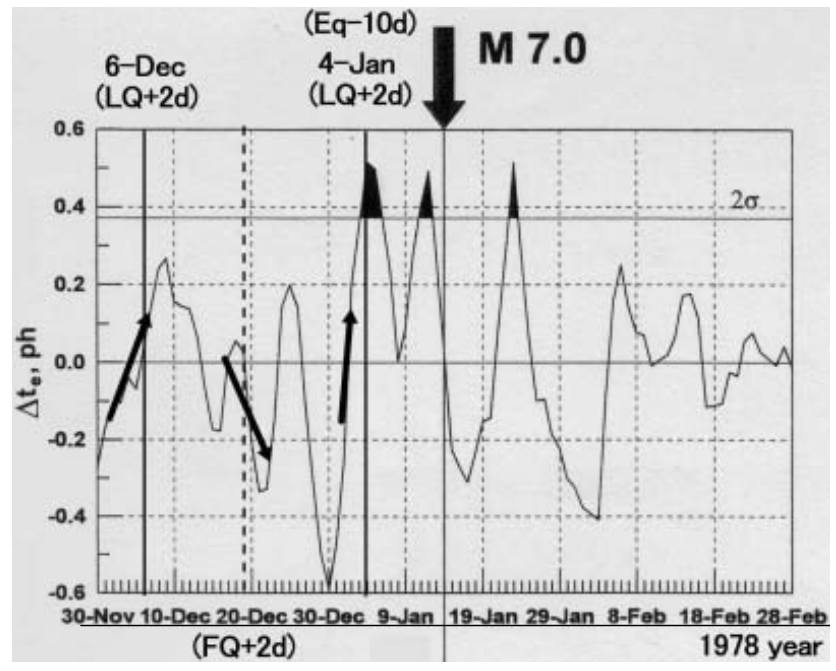

Fig. 6. Lunar phase analysis for $\Delta t e$ (phase) for the $1978 \mathrm{EQ}$ $(M=7.0)$.

pattern in this case is basically similar to that of the Kobe EQ shown in Fig. 5.

\section{Case 3: the 1980 earthquake $(M=6.1)$}

Figure 7 is the evening TT $(\Delta t e, p h)$ of the VLF propagation for this EQ. There is an anomaly which exceeded $2 \sigma$ before the EQ. And the anomaly is chosen as a reference point for the analysis. The anomaly appeared 3 days before the EQ and the day was a full moon - 2 day, which is shown as F-2d in the figure. The day of same lunar phase of one month before and the day between the two which is the day of new moon -2 day, were added in the figure. The visual inspection does not yield a clear correlation, but the FFT analysis indicates the periodicity of $\sim 30$ days, 14 days and 5 days, which might suggest the effect of earth tides.

\section{Case 4: the 1978 earthquake $(M=6.1)$}

Figure 8 is the temporal variation of the evening TT $(\Delta t e$, $p h$ ) of the VLF propagation for this EQ. There are anomalies which exceed $2 \sigma$ twice just before the EQ, and the first anomaly is chosen as the reference point for the analysis. The anomaly appeared 9 days before the main shock and the day was a last quarter moon -3 days (LQ-3d). The day of same lunar phase of one month before, and the day between the two which is the day of a first quarter moon -3 days were included in the figure. Since the pattern is basically same as those for Figs. 5 and 6, the detailed explanation is omitted. 


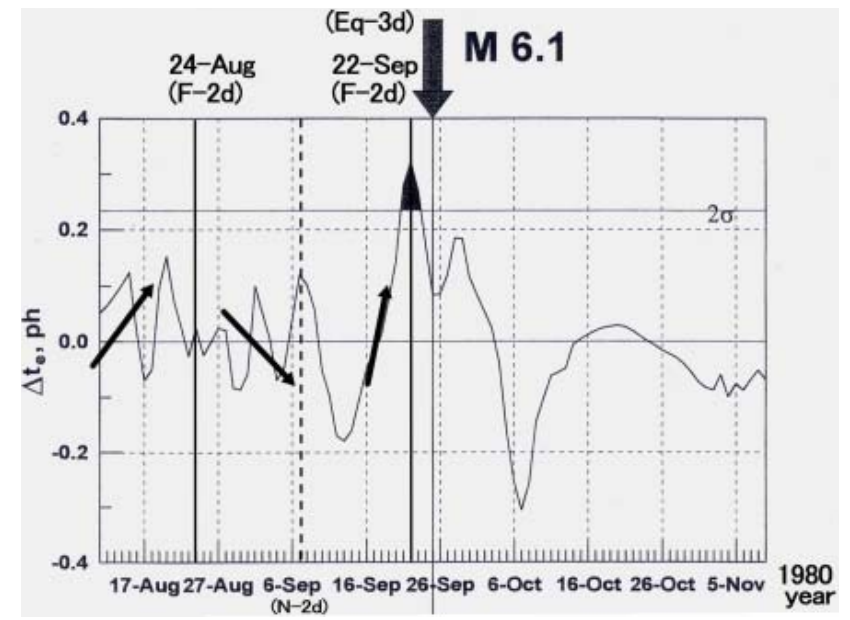

Fig. 7. Lunar phase analysis for $\Delta t e(\mathrm{ph})$ for the $1980 \mathrm{EQ}(M=6.1)$.

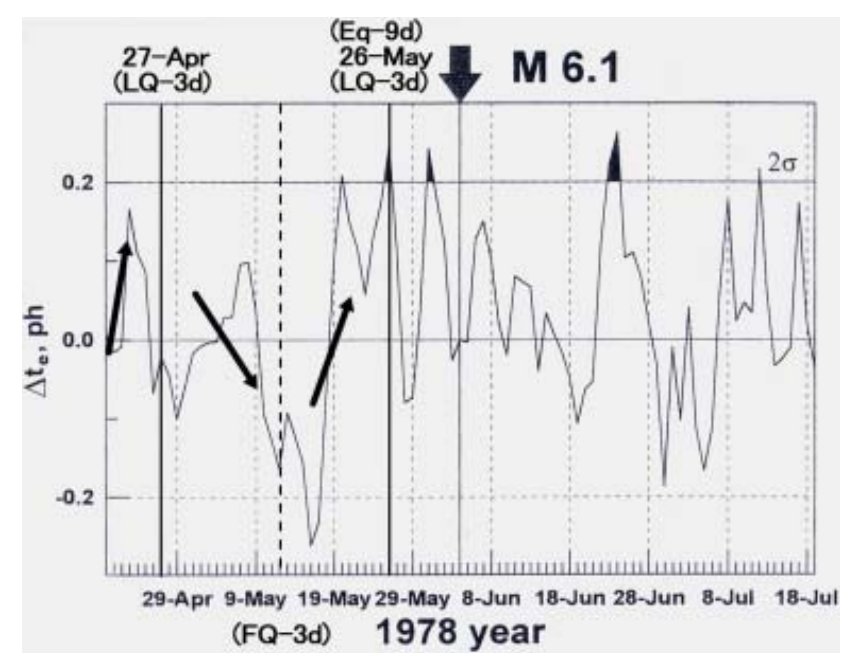

Fig. 8. Lunar phase analysis for $\Delta t e(\mathrm{ph})$ for the $1978 \mathrm{EQ}(M=6.1)$.

Cases 5 and 6: the 1990 earthquake $(M=6.4)$ and the 1989 earthquake $(M=6.0)$

These are the cases for which no anomalies were detected before the EQs in Fig. 9, which can be used to understand the background (or normal) situation. The observed patterns for these cases are the ones which do not show any maximumminimum-maximum pattern nor the $2 \sigma$ anomaly one month before the EQ. They are quite different from the cases explained above.

\subsection{Anomalies observed in other frequencies at the 1995 Kobe earthquake}

Many types of anomalies were reported for the Kobe EQ, and Fig. 10 is a summary of those different phenomena (Nagao

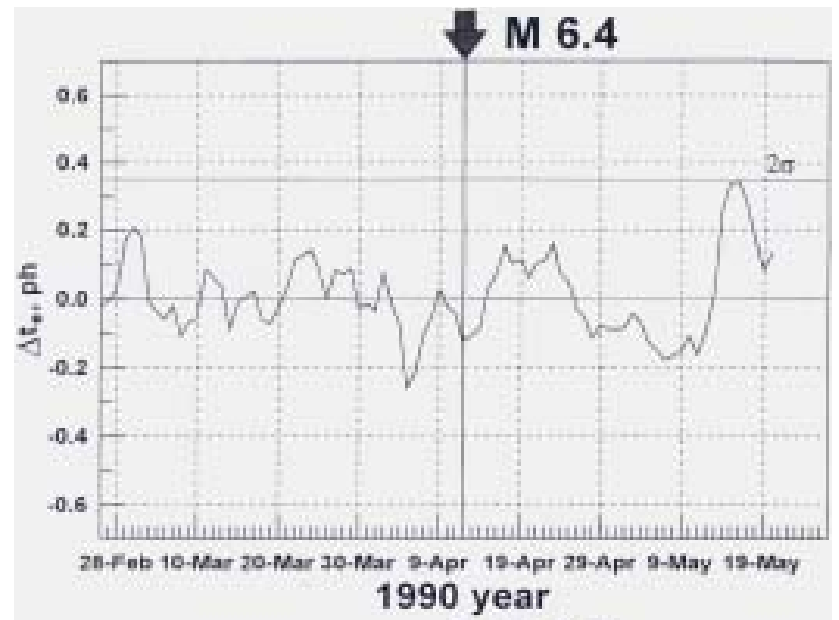

M 6.0

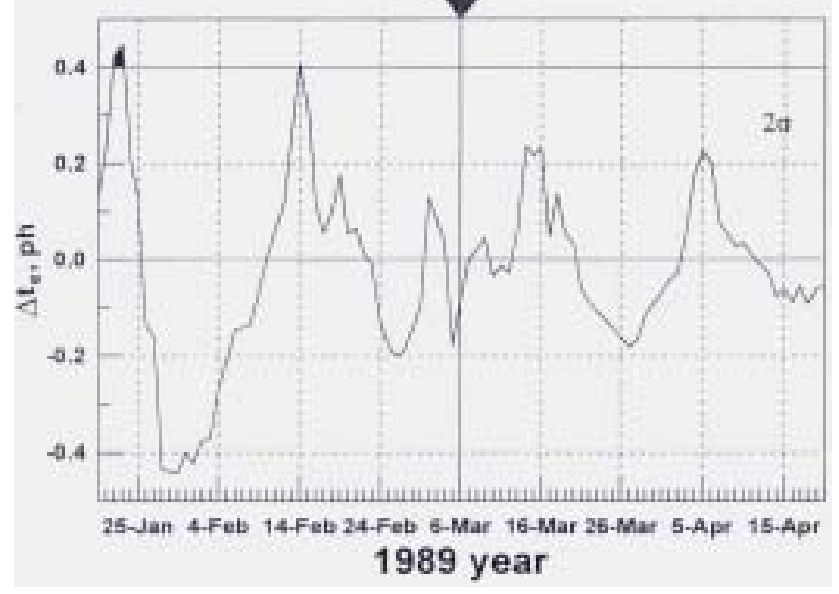

Fig. 9. Lunar phase analysis for $\Delta t e(\mathrm{ph})$ for the $1980 \mathrm{EQ}(M=6.4)$ and the 1989 EQ $(M=6.0)$.

et al., 2002). The figure shows that on 9 January 1995 that is 8 days before the EQ, anomalies of ELF, VLF, LF and HF radio emissions as well as atmospherics (indicated by lightning) were observed. These phenomena are indicated by a blue box, and they are thought as precursors to the EQ.

On 7 December 1994 that is one month before 9 January, there were also observed anomalies of VLF, LF and HF radio emissions as well as atmospherics. The first anomaly appeared on 9 January which corresponds to a first quarter moon. The same lunar phase day of one month before is 10 December. So the anomalies in December and January are found to have occurred under the similar lunar phases. Besides the measurements show a maximum-flat-maximum pattern, which is similar to the measurements of VLF in the previous subsection. 


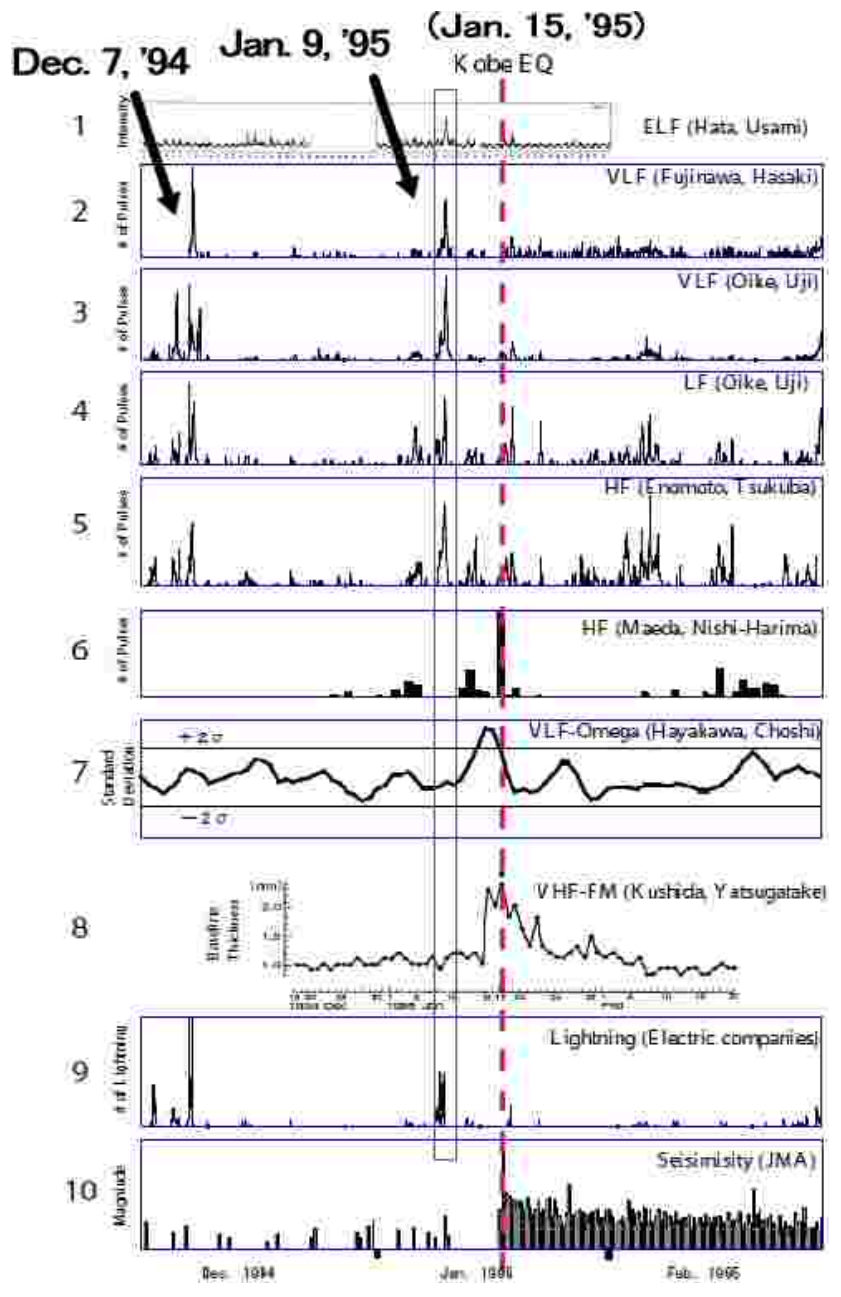

Fig. 10. Electromagnetic phenomena for the Kobe EQ (15 January 1995). Radio emissions at ELF (Row 1), ULF (2 and 3), LF (4), HF (5 and 6), subionospheric VLF (Omega(Tsushima)-Inubo) (7), over-horizon VHF (8), lightning (9) and seismicity (10). 9 January is an anomalous day (marked by a blue box).

\section{Conclusion and discussion}

We have found the first evidence on the effect of earth tides in the VLF/LF propagation anomaly for a particular huge EQ (named 2005 Miyagi-oki EQ) (Muto et al., 2009), so that we have tried to generalize this study to a much more data set. We have studied a few categories of seismogenic effects. First, ULF emissions from the lithosphere or the EQ focal region are treated, which are directly associated with the tectonic (or mechanical) effect (probably microscopic, not macroscopic) of the lithosphere. Secondly, we have tried to find our any tidal effects in the subionospheric VLF/LF propagation data indicating the seismo-ionospheric perturbations. Finally, we have examined the tidal effect in the overall observations for the Kobe EQ.
The following findings have emerged from this study.

1. ULF geomagnetic anomalies are found to show a maximum-minimum-maximum pattern in synchronism with the lunar phase of the EQ during several months before its occurence.

2. VLF/LF propagation anomalies are also found to exhibit the similar variation as that of ULF emissions, but not so clear as compared with the ULF emissions. Sometimes we have found some shift with the lunar phase and more irregularities in the variation. The deviation from the average value is found to increase with time, thus the peak before the EQ is the largest deviation usually exceeding $2 \sigma$. After this peak we observe some oscillations and there will be the occurrence of an EQ around the time when the deviation is just around zero.

3. Radio emissions at different frequencies (ULF, VLF, LF, VHF, etc.) are found to contain the similar tidal modulation.

The lunar tidal effect in EQ triggering is recognized recently in the field of seismology (Tanaka et al., 2004), in which this effect becomes very apparent only before a large EQ. Similarly, there have been accumulated a lot of evidences on the electromagnetic effects as EQ signatures, which are believed to be very complimentary to the EQ mechanical phenomena. The initial action of our seismogenic electromagnetic effects is definitely any mechanical effect in the lithosphere (or around the EQ focal zone), so that such a tidal effect might be involved even in the seismogenic electromagnetic effects when the lithosphere is just at a critical or at a super-critical stage. This is our initial motivation of the study.

According to Tanaka et al. (2004), the tidal effect is likely to be clearly seen for huge EQs (with larger magnitude) in which the focal zone is well self-organized to a critical or a super-critical stage. We have already known that both of ULF electromagnetic emissions and ionospheric perturbations as seen by VLF signals are only observed for huge EQs (at least with magnitude greater than 6.0 or so) (Hayakawa et al., 2007; Maekawa et al., 2006), so that we have analyzed our former data satisfying this EQ magnitude criterion in this analysis.

The lithospheric phenomenon of ULF emissions is found to exhibit a rather clear modulation of earth tides, which means that the ULF emissions observed for the 1993 Guam EQ and 2000 Izu EQ swarm are clearly modulated by earth tides one and two months before the EQ. This, in turn, leads to the idea that the presence of tidal modulation would be a very strong support to that those emissions are highly likely to be seismogenic and to be attributed to some effect like microfracturing (Molchanov and Hayakawa, 1995, 1998a) as the mechanical effect (though probably microscopic) in the lithosphere. We have already known that the lithosphere for 
a huge EQ is well self-organized up to a critical or a supercritical stage by means of former fractal analyses (Hayakawa et al., 1999; Smirnova et al., 2001, 2004; Kapiris et al., 2002; Gotoh et al., 2003, 2004; Ida et al., 2005, 2006; Hayakawa and Ida, 2008). Because the focal zone is well self-organized, we are ready to anticipate the generation of different kinds of seismogenic electromagnetic phenomena. Especially, the seismogenic ULF emission is the direct consequence of the lithosphere (or mechanical (microscopic microfracturing) effect), and this is the reason why we have observed a clear tidal effect. The identification of such a clear tidal effect might offer an important physical indication that those ULF emissions reported before are highly likely to be precursors of EQs.

On the other hand, the VLF/LF data suggesting the presence of seismo-ionospheric perturbations are found to show the similar tidal modulation. However, the effect is not so clear as compared with the case of ULF emissions in such a way that we sometimes find a certain shift with respect to the lunar phase, the presence of some higher frequency modulations. This is easy to understand because the modulation is not direct as in the case of ULF emissions, but it might propagate through the atmosphere and up to the ionosphere.

We believe that the study in this paper would offer a bridge between the seismological (or mechanical) effect and our seismo-electromagnetics effect. Also, the presence of tidal effect would be of essential importance in obtaining further confident confirmation of those effects seeming to be an EQ precursor. Of course, we have to mention that we have to increase the number of events large enough to persuade everybody.

Acknowledgements. A part of this study is supported by NICT, to which we are grateful.

Edited by: M. E. Contadakis

Reviewed by: P. F. Biagi and A. P. Nickolaenko

\section{References}

Fraser-Smith, A. C., Bernardi, A., McGill, P. R., Ladd, M. E., Helliwll, R. A., and Villard Jr., O. G.: Low-frequency magnetic field measurements near the epicenter of the Ms 7.1 Loma Preita earthquake, Geophys. Res. Lett., 17, 1465-1468, 1990.

Gotoh, K., Hayakawa, M., and Smirnova, N.: Fractal analysis of the ULF geomagnetic data obtained at Izu Peninsula, Japan in relation to the nearby earthquake swarm of June-August 2000, Nat. Hazards Earth Syst. Sci., 3, 229-236, 2003, http://www.nat-hazards-earth-syst-sci.net/3/229/2003/.

Gotoh, K., Hayakawa, M., Smirnova, N. A., and Hattori, K.: Fractal analysis of seismogenic ULF emissions, Phys. Chem. Earth, 29, 419-424, 2004.

Gotoh, K., Akinaga, Y., Hayakawa, M., and Hattori, K.: Principal component analysis of ULF geomagnetic data for Izu islands earthquakes in July 2000, Journal of Atmospheric Electricity, 22, $1-12,2002$.
Gufeld, I. L. and Marenko, V. F.: The short-time prediction for the time of strong earthquakes with the help of radiowave technique, Doklady RAN, 323, 1064-1070, 1992.

Gufeld, I. L., Gusev, G., and Pokhotelov, O.: Is the prediction of earthquake date possible by VLF radio wave monitoring method?, in: Electromagnetic Phenomena Related to Earthquake Prediction, edited by: Hayakawa, M. and Fujinawa, Y., Terra Sci. Publ. Comp., Tokyo, 381-390, 1994.

Hayakawa, M.: Electromagnetic Precursors of Earthquakes: Review of Recent Activities, Rev. Radio Sci. 1993-1996, edited by: Stone, W. R., Oxford Univ. Press, 807-818, 1997.

Hayakawa, M. (Ed.): Atmospheric and Ionospheric Electromagnetic Phenomena Associated with Earthquakes, Terra Sci. Pub. Co., Tokyo, 996 pp., 1999.

Hayakawa, M.: Electromagnetic phenomena associated with earthquakes: A frontier in terrestrial electromagnetic noise environment, Recent Res. Devel. Geophysics, 6, 81-112, 2004.

Hayakawa, M.: VLF/LF radio sounding of ionospheric perturbations associated with earthquakes, Sensors, 7, 1141-1158, 2007.

Hayakawa, M., Hattori, K., and Ohta, K.: Monitoring of ULF (ultralow-frequency) geomagnetic variations associated with earthquakes, Sensors, 7, 1108-1122, 2007.

Hayakawa, M. and Sato, H.: Ionospheric perturbations associated with earthquakes, as detected by subionospheric VLF propagation, in: Electromagnetic Phenomena Related to Earthquake Prediction, edited by: Hayakawa, M. and Fujinawa, Y., Terra Sci. Publ., Tokyo, 391-398, 1994.

Hayakawa, M. and Molchanov, O. A. (Eds.): Seismo Electromagnetics: Lithosphere-Atmosphere-Ionosphere Coupling, TERRAPUB, Tokyo, 477 pp., 2002.

Hayakawa, M. and Molchanov, O. A.: Seismo-electromagnetics as a new field of radiophysics: Electromagnetic phenomena associated with earthquakes, Radio Science Bull., 320, 8-17, 2007.

Hayakawa, M., Kawate, R., Molchanov, O. A., and Yumoto, K.: Results of ultra-low-frequency magnetic field measurements during the Guam earthquake of 8 August 1993, Geophys. Res. Lett., 23, 241-244, 1996a.

Hayakawa, M., Molchanov, O. A., Ondoh, T., and Kawai, E., The precursory signature effect of the Kobe earthquake on VLF subionospheric signals, J. Comm. Res. Lab., 43, 169-180, 1996b.

Hayakawa, M., Itoh, T., and Smirnova, N.: Fractal analysis of ULF geomagnetic data associated with the Guam earthquake on August 8, 1993, Geophys. Res. Lett., 26, 2797-2800, 1999.

Ida, Y. and Hayakawa, M.: Fractal analysis for the ULF data during the 1993 Guam earthquake to study prefracture criticality, Nonlin. Processes Geophys., 13, 409-412, 2006, http://www.nonlin-processes-geophys.net/13/409/2006/.

Kapiris, P., Polygiannakis, J., Peratzakis, A., Nomicos, K., and Eftaxias, K.: VHF-electromagnetic evidence of underlying preseismic critical stage, Earth Planets Space, 54, 1237-1246, 2002.

Kopytenko, Y. U. A., Matiashvili, T. G., Veronov, P. M., and Molchanov, O. A.: Detection of ultra-low frequency emissions connected with the Spitak earthquake and its aftershock activity, based on geomagnetic pulsation data at Dusheti and Vardzia, Phys. Earth Planet. In., 77, 85-95, 1993.

Maekawa, S., Horie, T., Yamauchi, T., Sawaya, T., Ishikawa, M., Hayakawa, M., and Sasaki, H.: A statistical study on the effect of earthquakes on the ionosphere, based on the subionospheric LF propagation data in Japan, Ann. Geophys., 24, 2219-2225, 
2006, http://www.ann-geophys.net/24/2219/2006/.

Molchanov, O. A. and Hayakawa, M.: Generation of ULF electromagnetic emissions by microfracturing, Geophys. Res. Lett., 22, 3091-3094, 1995.

Molchanov, O. A. and Hayakawa, M.: On the generation mechanism of ULF seismogenic electromagnetic emissions, Phys. Earth Planet. In., 105, 201-210, 1998a.

Molchanov, O. A. and Hayakawa, M.: Subionospheric VLF signal perturbations possibly related to earthquakes, J. Geophys. Res., 103(A8), 17489-17504, 1998b.

Molchanov, O. A. and Hayakawa, M.: Seismo Electromagnetics and Related Phenomena: History and latest results, TERRAPUB, Tokyo, 189 pp., 2008.

Molchanov, A., Hayakawa, M., Ondoh, T., and Kawai, E., Precursory effects in the subionospheric VLF signals for the Kobe earthquake, Phys. Earth Planet. In., 105, 239-248, 1998.

Muto, F., Kasahara, Y., Hobara, Y., Hayakawa, M., Rozhnoi, A., Solovieva, M., and Molchanov, O. A.: Further study on the role of atmospheric gravity waves on the seismo-ionospheric perturbations as detected by subionospheric VLF/LF propagation, Nat. Hazards Earth Syst. Sci., 9, 1111-1118, 2009, http://www.nat-hazards-earth-syst-sci.net/9/1111/2009/.

Nagao, T., Enomoto, Y., Fujinawa, Y., Hata, M., Hayakawa, M., Huang, Q., Izutsu, J., Kushida, Y., Maeda, K., Oike, K., Uyeda, S., and Yoshino, T.: Electromagnetic anomalies associated with 1995 Kobe earthquake, J. Geodyn., 33, 477-487, 2002.

Smirnova, N., Hayakawa, M., and Gotoh, K.: Precursory behavior of fractal characteristics of the ULF electromagnetic fields in seismic active zones before strong earthquakes, Phys. Chem. Earth, 29, 445-451, 2004.
Smirnova, N., Hayakawa, M., Gotoh, K., and Volobuev, D.: Scaling characteristics of ULF geomagnetic fields at the Guam seismoactive area and their dynamics in relation to the earthquake, Nat. Hazards Earth Syst. Sci., 1, 119-126, 2001, http://www.nat-hazards-earth-syst-sci.net/1/119/2001/.

Sue, Y.: The effect of earth tides in triggering earthquake as clearly observed in some specific regions of Japan, Journal of Atmospheric Electricity, 29, 53-62, 2009.

Tanaka, S.: Tidal triggering of earthquakes precursory to the 2004 $\mathrm{Mw}=9.0$ off Sumatra earthquake, Paper presented at 4th International Workshop on Statistical Seismology, Graduate Univ. for Advanced Study, Kanagawa, Japan, 9-13 January 2006.

Tanaka, S., Ohtake, M., and Sato, H.: Tidal triggering of earthquakes in Japan related to the regional tectonic stress, Earth Planets Space, 56(5), 511-515, 2004.

Toda, S., Stein, R. S., and Sagiya, T.: Evidence from the AD 2000 Izu islands swarm that stressing rate governs seismicity, Nature, 419, 58-61, 2002.

Uyeda, S., Hayakawa, M., Nagao, T., Molchanov, O., Hattori, K., Orihara, Y., Gotoh, K., Akinaga, Y., and Tanaka, H.: Electric and Magnetic phenomena observed before the volcano-seismic activity in 2000 in the Izu Island Region, Japan, P. Natl. Acad. Sci. USA (PNAS), 99(11), 7352-7355, 2002

Yoshida, M., Yamauchi, T., Horie, T., and Hayakawa, M.: On the generation mechanism of terminator times in subionospheric VLF/LF propagation and its possible application to seismogenic effects, Nat. Hazards Earth Syst. Sci., 8, 129-134, 2008, http://www.nat-hazards-earth-syst-sci.net/8/129/2008/. 